\title{
TUNNELING MAGNETORESISTANCE IN PLANAR FERROMAGNETIC JUNCTIONS
}

\author{
M. WILCZYŃSKI AND J. BARNAŚ \\ ${ }^{a}$ Institute of Physics, Warsaw Technical University \\ Koszykowa 75, 00-662 Warszawa, Poland \\ ${ }^{b}$ Department of Physics, Adam Mickiewicz University \\ Umultowska 85, 61-614 Poznań, Poland
}

\begin{abstract}
Bias dependence of the tunnel magnetoresistance in simple planar ferromagnetic junctions is considered theoretically within the one-band model. The limit of sequential tunnelling in double junctions with a non-magnetic central electrode is studied as well. In this case tunnel magnetoresistance exists only when the spin relaxation time due to spin-flip scattering processes inside the central electrode is sufficiently long.
\end{abstract}

PACS numbers: 72.15.Gd, 73.40.Gk, 75.70.-i

The tunnel magnetoresistance (TMR) effect is known since the first experimental observation by Julliere [1], who found a change of nearly 14 per cent at $T=4.2 \mathrm{~K}$ in the tunnel conductance in $\mathrm{Co} / \mathrm{Ge} / \mathrm{Fe}$ junctions, when the magnetic moments of ferromagnetic electrodes rotated from antiparallel to parallel alignment. The effect is usually described by the ratio $\Delta R / R_{\mathrm{P}} \equiv\left(R_{\mathrm{AP}}-R_{\mathrm{P}}\right) / R_{\mathrm{P}}$, where $R_{\mathrm{AP}}$ and $R_{\mathrm{P}}$ are the junction resistances in the antiparallel and parallel magnetic configurations, respectively. Similar effect was also reported in other ferromagnetic junctions [2], and recent experimental data show additionally that TMR significantly decreases with increasing bias voltage [3].

In this paper we consider TMR within the spin-polarised free-electron-like one-band model. The zero bias limit, $V=0$, of this model was studied by Slonczewski [4]. Here, we extend his description to a finite voltage by taking into account the bias dependence of the barrier shape and height. The description is also extended to double junctions, in which both external electrodes are ferromagnetic, while the central electrode is non-magnetic. Accordingly, we assume a rectangular barrier at $V=0$, which changes into a trapezoidal one when a bias voltage $V$ is applied. Let us consider first the transmission probability for an electron of energy $\epsilon$ and spin $\sigma(\sigma=\uparrow, \downarrow)$, incident normally on the barrier, and assume that tunnelling processes conserve electron spin. In strictly parallel or antiparallel configurations electrons with opposite spins can be considered separately and the tunnelling probability is then determined by a solution of the one-dimensional Schrödinger equation. From the continuity conditions of the electron wave function 
and its first derivative at the electrode/barrier interfaces one finds the following transmission coefficient for the $\mu$-th configuration $(\mu=\mathrm{AP}, \mathrm{P})[5]$ :

$$
T_{\sigma, \mu}(\epsilon)=\frac{4 k_{3 \sigma, \mu}}{\pi^{2} k_{1 \sigma}}\left(M_{\sigma, \mu}^{2}+N_{\sigma, \mu}^{2}\right)^{-1},
$$

where (for the electrodes made of the same ferromagnet)

$$
\begin{aligned}
M_{\sigma, \mu} & =\frac{D}{k_{1 \sigma}}\left[\mathrm{Ai}^{\prime}\left(z_{0}\right) \mathrm{Bi}^{\prime}\left(z_{d}\right)-\mathrm{Ai}^{\prime}\left(z_{d}\right) \mathrm{Bi}^{\prime}\left(z_{0}\right)\right] \\
& +\frac{k_{3 \sigma, \mu}}{D}\left[\mathrm{Ai}\left(z_{0}\right) \operatorname{Bi}\left(z_{d}\right)-\operatorname{Ai}\left(z_{d}\right) \operatorname{Bi}\left(z_{0}\right)\right], \\
N_{\sigma, \mu} & =\frac{k_{3 \sigma, \mu}}{k_{1 \sigma}}\left[\operatorname{Ai}\left(z_{d}\right) \mathrm{Bi}^{\prime}\left(z_{0}\right)-\operatorname{Ai}^{\prime}\left(z_{0}\right) \operatorname{Bi}\left(z_{d}\right)\right] \\
& +\left[\operatorname{Ai}\left(z_{0}\right) \operatorname{Bi}^{\prime}\left(z_{d}\right)-\mathrm{Ai}^{\prime}\left(z_{d}\right) \operatorname{Bi}\left(z_{0}\right)\right], \\
k_{1 \sigma}= & \frac{1}{\hbar} \sqrt{2\left(\epsilon+p_{\sigma} \Delta\right) m}, \\
k_{3 \sigma, \mu} & =\frac{1}{\hbar} \sqrt{2\left(\epsilon+p_{\sigma} p_{\mu} \Delta+e V\right) m .} .
\end{aligned}
$$

In the above equations $\operatorname{Ai}(z)$ and $\operatorname{Bi}(z)$ are the Airy functions, $\operatorname{Ai}^{\prime}(z)$ and $\operatorname{Bi}^{\prime}(z)$ are their first derivatives, $2 \Delta$ denotes spin splitting of the electron band, $p_{\sigma}=1$ $\left(p_{\sigma}=-1\right)$ for $\sigma=\uparrow(\sigma=\downarrow), p_{\mu}=1\left(p_{\mu}=-1\right)$ for $\mu=\mathrm{P}(\mu=\mathrm{AP})$, and $m$ denotes the electron mass. Apart from this, the following definitions have been introduced:

$$
\begin{aligned}
& z_{0}=(\sqrt{2 m} d / e V \hbar)^{2 / 3}\left(E_{\mathrm{F}}+U-\epsilon\right), \\
& z_{d}=(\sqrt{2 m} d / e V \hbar)^{2 / 3}\left(E_{\mathrm{F}}+U-\epsilon-e V\right), \\
& D=-\left(2 m e V / \hbar^{2} d\right)^{1 / 3},
\end{aligned}
$$

where $E_{\mathrm{F}}$ is the Fermi energy measured from the middle point between the edges of the two spin subbands, $U$ is the barrier height measured from the Fermi level and $-e$ is the electron charge.

Having found the transmission coefficient one can calculate the tunneling current. In real systems there are many electrons incident on the barrier from both sides and the net current is a sum of contributions from all tunnelling processes [6]. When the electrode/barrier interfaces are flat, the in-plane component $k_{\|}$of the electron wave vector $k$ is conserved in tunnelling events. At $T=0$ the net electric current per unit area can be then calculated from the formula

$$
j_{\mu}=\frac{2 \pi m e}{h^{3}} \sum_{\sigma}\left[e V \int_{E_{0 \sigma \mu}}^{E_{\mathrm{F}}-e V} T_{\sigma, \mu}(\epsilon) \mathrm{d} \epsilon+\int_{E_{\mathrm{F}}-e V}^{E_{\mathrm{F}}}\left(E_{\mathrm{F}}-\epsilon\right) T_{\sigma, \mu}(\epsilon) \mathrm{d} \epsilon\right],
$$


where $\epsilon$ is now the energy associated with normal motion of the electron, whereas $E_{0 \sigma \mu}=-p_{\sigma} \Delta$ for the parallel configuration $(\mu=\mathrm{P})$ and $E_{0 \sigma \mu}=\Delta-\delta_{\sigma, \uparrow} \mathrm{eV}$ for the antiparallel configuration $(\mu=\mathrm{AP})$. The junction resistance can be then calculated as $R_{\mu}=V / j_{\mu} A$ ( $A$ is the junction area), and TMR as $\Delta R / R_{\mathrm{P}}$.

In Fig. 1 we show TMR as a function of the bias voltage. For the parameters assumed there TMR decreases with increasing $V$. This behaviour of TMR, however, is not universal within the model considered here. An opposite behaviour, i.e. increasing TMR with increasing voltage, occurs in a certain range of small barrier heights.
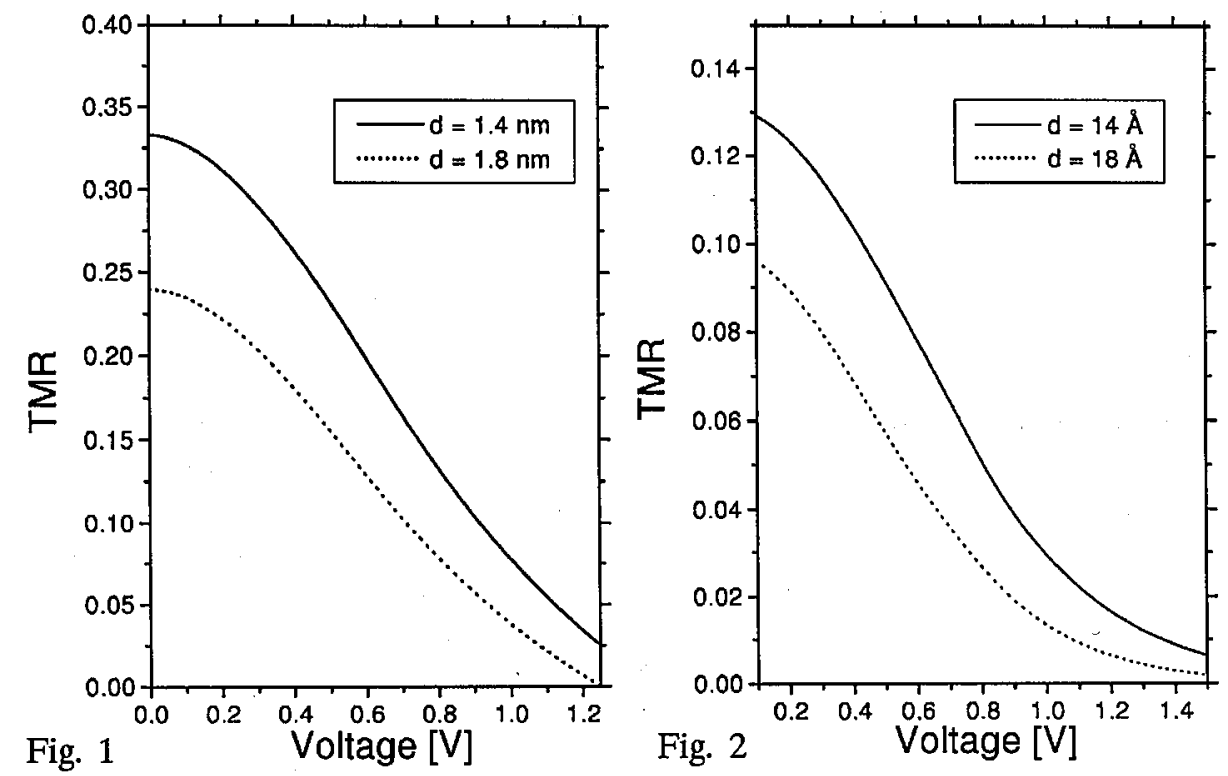

Fig. 1. Bias dependence of TMR in a single planar junction for two different thicknesses of the barrier. The other parameters assumed here are: $E_{\mathrm{F}}=2 \mathrm{eV}, U=3 \mathrm{eV}$, $\Delta=1.5 \mathrm{eV}$, and $d$ as indicated.

Fig. 2. Bias dependence of TMR in a double planar junction with a non-magnetic central electrode and with two identical barriers, calculated in the limit of infinite spin relaxation time. The other parameters assumed here are: $E_{\mathrm{F}}=2 \mathrm{eV}, U=3 \mathrm{eV}$, $\Delta=1.5 \mathrm{eV}$, and $d$ as indicated.

Let us consider now tunnelling in a double junction with a non-magnetic central electrode. In the sequential tunnelling limit the electron tunnelling from the source to sink electrodes is a two-stage process; in the first stage a given electron tunnels through one of the barriers, while in the second stage another electron tunnels through the second barrier. For simplicity we neglect quantization of the energy levels in the central electrode. There is no TMR in the limit of fast energy and spin relaxations. When, however, the spin relaxation time inside the central electrode is long, then the spin asymmetry in tunnelling rates through the barriers gives rise to some spin accumulation in the central electrode. In that limit electric 
current is conserved for each spin channel separately. Spin accumulation depends on the magnetic configuration of the junction, and this dependence can lead to TMR. In Fig. 2 we show the bias dependence of TMR in the limit of infinite spin relaxation time inside the non-magnetic central electrode. As before, the effect decreases with increasing voltage.

\section{Acknowledgments}

This work was supported through the Research Project 8T11F02716 of the Committee for Scientific Research.

\section{References}

[1] M. Julliere, Phys. Lett. A 54, 225 (1975).

[2] T. Miyazaki, T. Yaoi, S. Ishio, J. Magn. Magn. Mater. 98, L7 (1991); J. Nowak, J. Raułuszkiewicz, J. Magn. Magn. Mater. 109, 79 (1992).

[3] J.S. Moodera, L.R. Kinder, T.M. Wong, R. Meservey, Phys. Rev. Lett. 74, 3273 (1995); J.S. Moodera, L.R. Kinder, J. Appl. Phys. 79, 4724 (1996).

[4] J.C. Slonczewski, Phys. Rev. B 39, 6995 (1989).

[5] K.H. Gundlach, Solid-State Electron. 9, 949 (1966).

[6] J.G. Simmons, J. Appl. Phys. 34, 1793 (1963). 